\title{
Diacronie
}

Studi di Storia Contemporanea

$\mathrm{N}^{\circ} 10,2 \mid 2012$

Digital History: la storia nell'era dell'accesso

\section{Navigare la storia: considerazioni sulla creazione di un portale di storia ambientale}

Wilko Graf von Hardenberg e Kimberly Coulter

\section{(C) OpenEdition}

\section{Journals}

\section{Edizione digitale}

URL: http://journals.openedition.org/diacronie/2867

DOI: $10.4000 /$ diacronie. 2867

ISSN: 2038-0925

Editore

Association culturelle Diacronie

Notizia bibliografica digitale

Wilko Graf von Hardenberg e Kimberly Coulter, « Navigare la storia: considerazioni sulla creazione di un portale di storia ambientale », Diacronie [Online], № 10,2 | 2012, documento 8, Messo online il 29 juin 2012, consultato il 06 mai 2019. URL : http://journals.openedition.org/diacronie/2867 ; DOI : 10.4000/diacronie.2867 


\title{
Diacronie
}

\section{8/}

\section{Navigare la storia: considerazioni sulla creazione di un portale di storia ambientale ${ }^{1}$}

\author{
Wilko GRAF VON HARDENBERG e Kimberly COULTER *
}

Questo articolo presenta l'esperienza dell'Environment \& Society Portal (www.environmentandsociety.org), uno dei più importanti progetti del Rachel Carson Center for Environment and Society di Monaco di Baviera*, online dai primi del 2012. Il portale intende rendere disponibili alla comunità accademica e al pubblico saggi originali, informazioni di carattere enciclopedico, mostre virtuali, libri, articoli, fonti e materiali multimediali nel campo delle cosiddette environmental humanities.

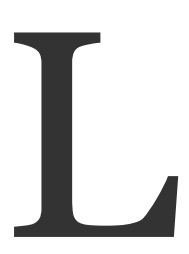

e environmental humanities sono un campo di indagine scientifica molto ampio e in rapida crescita che si propone di analizzare in modo olistico i rapporti tra natura e società e i modi in cui l'umanità interpreta l'ambiente naturale. Dato che non sarebbe possibile creare una collezione in grado di includere tutto il materiale prodotto in un campo così vasto, il progetto dell'Environment \& Society Portal va inteso piuttosto come un "gabinetto delle curiosità" dinamico ed interattivo, in costante crescita e che permetta agli utenti di reperire facilmente materiale di interesse. Nella raccolta dei contenuti l'attenzione si è dunque concentrata su singoli eventi e processi ritenuti particolarmente significativi e caratteristici, in grado insomma di riflettere in maniera esemplare le reciproche influenze tra umanità e ambiente naturale e di stimolare la riflessione sull'attuale questione ambientale. La sostenibilità dal punto di vista dei contenuti, ovvero la possibilità di avere sempre a disposizione materiale nuovo da mettere online è garantita dalla collaborazione e dai suggerimenti dei fellows internazionali del Carson Center, di partner istituzionali, e, in maniera crescente, della comunità online. 
Un portale umanistico online offre al pubblico la possibilità, ad integrazione della ricca offerta di musei e archivi, di scoprire ed esaminare nuove risorse e di condividere in maniera semplice ed intuitiva le proprie esperienze di ricerca con altri. E tutto questo senza che vi sia la necessità di allontanarsi dal proprio computer. Un portale di questo tipo contribuisce a superare confini sia disciplinari che nazionali, facilita l'accesso a tematiche complesse e stimola gli utenti a partecipare in maniera interattiva. Per venire incontro a queste esigenze nel modo migliore e per essere qualcosa in più di un mero contenitore di dati, un sito come l'Environment \& Society Portal deve essere in grado di offrire contenuti che siano al contempo accessibili e scientificamente validi, offrendo anche nuovi modi di collegare le informazioni tra loro e nuove prospettive di analisi. Un ulteriore obiettivo è quello di stimolare la discussione e l'incontro di idee, dentro e fuori dalle università, una necessità sempre più impellente in un campo d'indagine così ampio come la ricerca umanistica.

La messa a disposizione dei propri contenuti in maniera libera e gratuita è al centro della mission del portale. L'offerta non si limita solo a materiale originale prodotto per l'ambito digitale, ma include anche documenti digitalizzati espressamente per il portale. ${ }^{1}$ In vista di un crescente coinvolgimento degli utenti si stanno inoltre sviluppando strumenti che permettano, pur all'interno di una pubblicazione online edita e molto curata, di facilitare l'interazione con il pubblico, la conservazione delle ricerche svolte e la diffusione delle informazioni, oltre ad incoraggiare l'invio di contributi da parte degli utenti. L'offerta di contenuti multimediali mira ad essere la più varia possibile sia dal punto di vista della distribuzione geografica che da quello disciplinare. L’idea è che gli utenti non visitino un solo articolo o contributo multimediale, ma si trovino piuttosto di fronte ad uno strumento che gli permetta di intraprendere la propria personale "esplorazione".

Parti di questo articolo verranno presentate dagli autori ad Amburgo nel luglio 2012 in occasione della conferenza Digital Humanities 2012, mentre altre sono ispirate all'articolo già pubblicato in tedesco da Kimberly Coulter, «Digitale Erkundungen der Umwelt», in Kultur und Technik, 2/2012. Tutti i siti citati sono stati consultati il 22 aprile 2012.

* Il Rachel Carson Center è un istituto di ricerca della Ludwig-Maximilians-Universität e del Deutsches Museum di Monaco, generosamente finanziato dal Ministero Federale per l'Educazione e la Ricerca tedesco, che si occupa di promuovere la ricerca e la discussione nel campo dei rapporti tra società e ambiente e di rafforzare il ruolo delle scienze umane nell'attuale dibattito politico e scientifico sull'ambiente. Per ulteriori informazioni sul Rachel Carson Center si veda il sito:

URL: < http://www.rachelcarsoncenter.de > [consultato il 22 aprile 2012].

${ }^{1}$ Per quanto riguarda la digitalizzazione di libri e parti di libri il portale lavora in collaborazione con la Bayerische Staatsbibliothek di Monaco di Baviera, che ha una pluriennale esperienza nella digitalizzazione di materiale cartaceo, anche nel quadro di un accordo con Google Books 


\section{Condivisione e web annotation}

Uno dei problemi maggiori posti da un'impresa come questa, soprattutto in relazione alla retro-digitalizzazione di materiale a stampa, video e fotografie, è la questione dei diritti d'autore. Discernere tra le minuzie della legislazione sul copyright, soprattutto in un contesto internazionale, e prendere decisioni informate sulla possibilità o meno di pubblicare il materiale è un lavoro che prende molte energie $\mathrm{e}$ tempo. Siamo però convinti che sia un lavoro che ripagherà: la messa a disposizione solo di documenti di cui siano chiaramente definiti i modi in cui possono essere riutilizzati dà, infatti, la certezza di rendere il portale sostenibile nel lungo periodo e di contribuire al costante ricircolo delle informazioni. Proprio per agevolare la diffusione dei contenuti pubblicati sul sito abbiamo dunque deciso di corredare tutto il materiale prodotto espressamente per il portale con una licenza Creative Commons Attribuzione - Non commerciale - Condividi allo stesso modo ${ }^{2}$. Questa scelta è stata fatta anche per agevolare la possibile traduzione dei contenuti in altre lingue da parte degli utenti, il riutilizzo dei materiali pubblicati in contesti diversi e con ciò una loro maggiore diffusione3. Sempre in quest'ottica il sito offre la possibilità di condividere i contenuti attraverso un'ampia scelta di social media4. Sono poi allo studio opzioni che permettano di attivare un dialogo con e tra gli utenti a riguardo di particolari contenuti, sotto forma di commenti o altre soluzioni ancora allo studio, e di integrare l'Environment \& Society Portal con altri database presenti in rete, aumentando sempre di più la quantità di contenuti disponibili ${ }^{5}$. Una delle idee al vaglio del gruppo di lavoro è di implementare una soluzione che permetta la cosiddetta web annotation, ovvero la possibilità di aggiungere informazioni ai contenuti di un sito senza modificare la risorsa

${ }^{2}$ I dettagli della licenza sono disponibili a questo indirizzo:

URL: < http://creativecommons.org/licenses/by-nc-sa/3.o/ > [consultato il 22 aprile 2012].

3 Anche per quanto riguarda le immagini che corredano i testi, nella maggior parte dei casi si è preferito ricorrere, in modo da permettere agli utenti di condividere agevolmente anche queste, a materiale dotato di una qualunque licenza Creative Commons o che fossero nel pubblico dominio. Ci sono però ovviamente delle eccezioni, come nel caso del materiale messo a disposizione da alcuni partner istituzionali, come la Newberry Library di Chicago, per le quali non è stato possibile offrire licenze che permettano la ridistribuzione immediata del materiale.

4 È virtualmente possibile condividere i contenuti attraverso tutti i social media esistenti, grazie all'interfaccia offerta dal servizio AddThis. URL: < http://www.addthis.com > [consultato il 22 aprile 2012].

5 Per esempio l'Encyclopedia of Life URL: < http://www.eol.org > [consultato il 22 aprile 2012]; la Biodiversity Heritage Library URL: < http://www.biodiversitylibrary.org > [consultato il 22 aprile 2012]; o il database Natural Hazards del National Geophysical Data Center statunitense, URL: < http://www.ngdc.noaa.gov > [consultato il 22 aprile 2012]. 
originaria. Questo tipo di annotazioni si presenterebbero come un layer sovrimpresso ai contenuti, visibile ad altri utenti che usano lo stesso sistema di annotazione. In questo modo si potrebbe permettere agli utenti registrati di valutare e discutere pubblicamente la qualità dei contenuti, anche a livello di singoli paragrafi, con l'aggiunta di note a margine virtuali. Inoltre agli utenti verrebbe offerta la possibilità di migliorare i contenuti, secondo la filosofia del crowdsourcing, mantenendo però nettamente separata, in contrasto con il modello di Wikipedia, la dimensione di peer review sociale dal complesso processo editoriale e di validazione dei contenuti svolto dal team e dai collaboratori esterni del Rachel Carson Center. La speranza è che un modello di questo tipo possa in futuro combinare il meglio dei due mondi (crowdsourcing e lavoro editoriale tradizionale), offrendo contenuti interattivi che rispettino i più alti standard accademici6.

\section{Dalla ricerca mirata alla serendipità}

Con la crescita dei dati e delle fonti disponibili in formato digitale, sta crescendo anche il numero di modi in cui queste informazioni vengono presentate e gestite: la complessità e il livello di interrelazione delle modalità di ricerca online sta diventando sempre più grande, dando forma al contempo ad innovativi percorsi di analisi. D'altro canto però l'immediatezza ed apparente semplicità di una ricerca su Google rischia spesso di ridurre le possibilità di scoprire collegamenti e contenuti inaspettati o sorprendenti. Insomma, mentre crescono le nostre possibilità di trovare facilmente tutto il materiale relativo ad uno specifico argomento, allo stesso tempo ciò che non ricade intuitivamente nel campo della nostra stringa di ricerca rischia di diventare introvabile. Infatti, per fare una semplice ricerca su un motore di ricerca è necessario sapere esattamente cosa si sta cercando, ma l'approccio umanistico spesso parte invece dall'uso di metodologie più incentrate sull'esplorazione - come la funzione di browsing offerta da molti siti come JStor ${ }^{7}$ - e sulla concettualizzazione. In altre parole, nel mondo digitale si rischia di non avere più a disposizione quella dose di casualità produttiva che caratterizza tipicamente la ricerca in archivio o in biblioteca (il libro sconosciuto in una biblioteca a scaffale aperto, il documento importante nascosto in un

\footnotetext{
${ }^{6}$ Per un'analisi più dettagliata della social peer review e del suo possibile ruolo nel campo delle scienze umane si veda CASSELLA, Maria, "Social peer-review e scienze umane, ovvero 'della qualità nella Repubblica della scienza'», in JLIS.it, 1, 1/2010), DOI: 10.4403/jlis.it-30. Uno dei progetti più promettenti in questo campo è hypothes.it, che si basa sugli standard definiti dal consorzio Open Annotation Collaboration. URL: < http://openannotation.org > [consultato il 22 aprile 2012].

7 URL: < http://www.jstor.org > [consultato il 22 aprile 2012].
} 
faldone apparentemente banale). Si presenta dunque come una necessità, nell'ambito dell'umanistica digitale, andare oltre la semplice ricerca, integrandola con aspetti metodologici derivati dalle pratiche tradizionali dello storico ${ }^{8}$. Diventa cioè sempre più necessario tenere in considerazione che i nuovi strumenti digitali non aiutano solo a trovare quello che cerchiamo, ma che danno anche forma ai nostri percorsi di ricerca e, indirettamente, al tipo di risultati che possiamo ottenere. È perciò importante prendere attentamente in considerazione sia le strutture ontologiche che stanno dietro agli strumenti che usiamo (cosa esiste e come può essere catalogato?) sia i loro effetti a livello epistemologico (in che modo diversi tipi di classificazione e rappresentazione influenzano il nostro modo di interpretare il mondo?). È possibile conservare in ambito digitale quello spirito di serendipità e scoperta che caratterizza la ricerca storica? La consapevolezza dell'importanza di queste domande è stata alla base del processo all'interno del quale abbiamo sviluppato gli strumenti di ricerca e navigazione dell'Environment \& Society Portal. La nostra speranza è che l'uso di strumenti digitali sviluppati tenendo in considerazione le peculiarità dell'approccio umanistico sia in grado di generare risultati inaspettati e produrre nuovi punti di vista. Il portale, come vedremo nel dettaglio più avanti, mette dunque al centro della propria esperienza proprio la possibilità di permettere all'utente di fare scoperte inaspettate, sulla base dell'integrazione di ricerca geografiche, cronologiche e concettuali.

\section{L'Environment \& Society Portal: internazionalizzazione e interdisciplinarietà}

L’Environment \& Society Portal rappresenta un elemento centrale della strategia del Rachel Carson Center mirata a favorire il rafforzamento dell'internazionalizzazione e della messa in rete delle scienze umane. A questo si affianca l'obiettivo di stimolare in maniera decisa l'avvicinamento tra scienze umane e sociali da una parte e scienze naturali ed esatte dall'altra. Al centro dell'attività di raccolta del materiale vi sono dunque, ovviamente, i sei temi di ricerca istituzionali del Rachel Carson Center: catastrofi naturali e culture del rischio; saperi ambientali e società della conoscenza; mutamenti del paesaggio; consumo delle risorse e tutela della natura; imperialismo ecologico; etica, politica e movimenti ambientali. Il portale, sviluppato e pensato in

\footnotetext{
${ }^{8}$ Si veda a questo proposito il post di Friedel Grant sul blog delle Europeana Libraries «10 Things That Humanities Researchers Want», pubblicato il 18 aprile 2011, URL: < http://www.europeana-libraries.eu/home/-/blogs/10-things-that-humanities-researchers-want $>$ [consultato il 22 aprile 2012].
} 
inglese per facilitare la sua diffusione a livello internazionale, pubblica però anche materiale in altre lingue, corredato da un abstract in lingua inglese in modo da permettere a tutti di avere almeno un'idea dei contenuti offerti da ciascun documento. La raccolta e la cura dei contenuti del portale è affidata a un piccolo gruppo di lavoro presso il Rachel Carson Center. Questo gruppo internazionale, i cui membri sono qualificati nei più diversi campi delle scienze umane - dalla storia alla geografia passando per le scienze culturali - si avvale inoltre, a seconda dei bisogni della consulenza esterna, di ricercatori di caratura internazionale e del personale delle istituzioni partner. Dal punto di vista tecnico, grazie all'impegno di uno sviluppatore del software interno al progetto e ad alcuni consulenti esterni, il portale utilizza il Content Management System Drupal, che permette la più ampia libertà nella costruzione del sito e nell'integrazione di altri software liberi, a seconda di bisogni in continua evoluzione con la crescita dei contenuti e con il desiderio di coinvolgere sempre di più gli utenti esterni nel processo di creazione del sito.

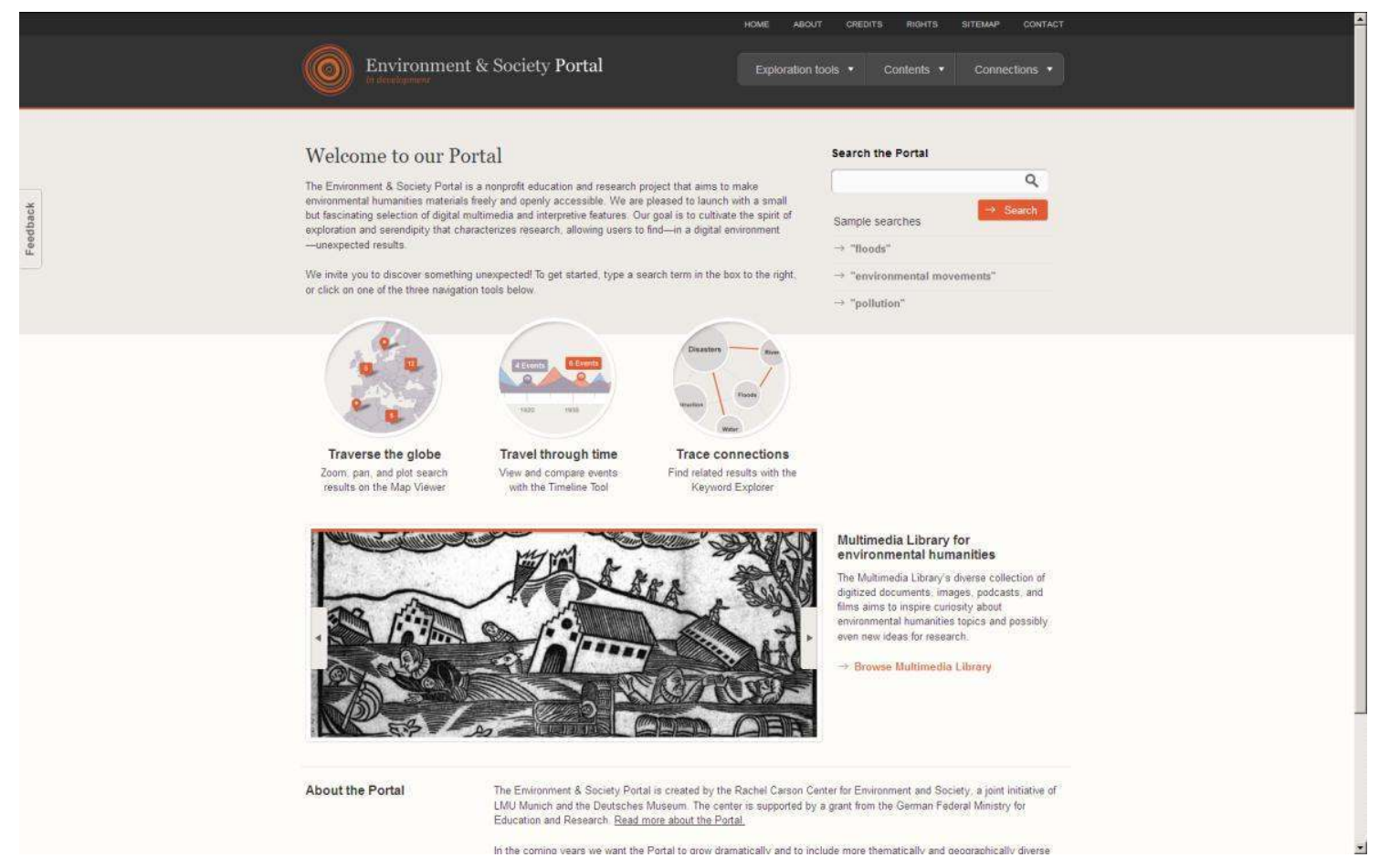

\subsection{I contenuti}

I curiosi avranno molte sorprese: nella biblioteca multimediale del portale si trovano i contenuti più diversi, che vanno da un podcast sul disastro di Fukushima ad articoli scientifici tratti da riviste di settore, contributi video e fonti primarie (per esempio una stampa del diciassettesimo secolo che rappresenta le alluvioni del Reno e 
dell'Elba). I ricercatori poi possono comparare diacronicamente e sincronicamente e mettere in relazione tra loro le brevi descrizioni di eventi storici significativi dal punto di vista ambientale. Per chi fosse interessato poi in analisi significative e fortemente localizzate di eventi e processi nella storia ambientale europea sono disponibili i brevi, ma accurati, articoli del sub-progetto Arcadia: European Environmental Histories ${ }^{9}$, curato assieme alla European Society for Environmental History¹0.

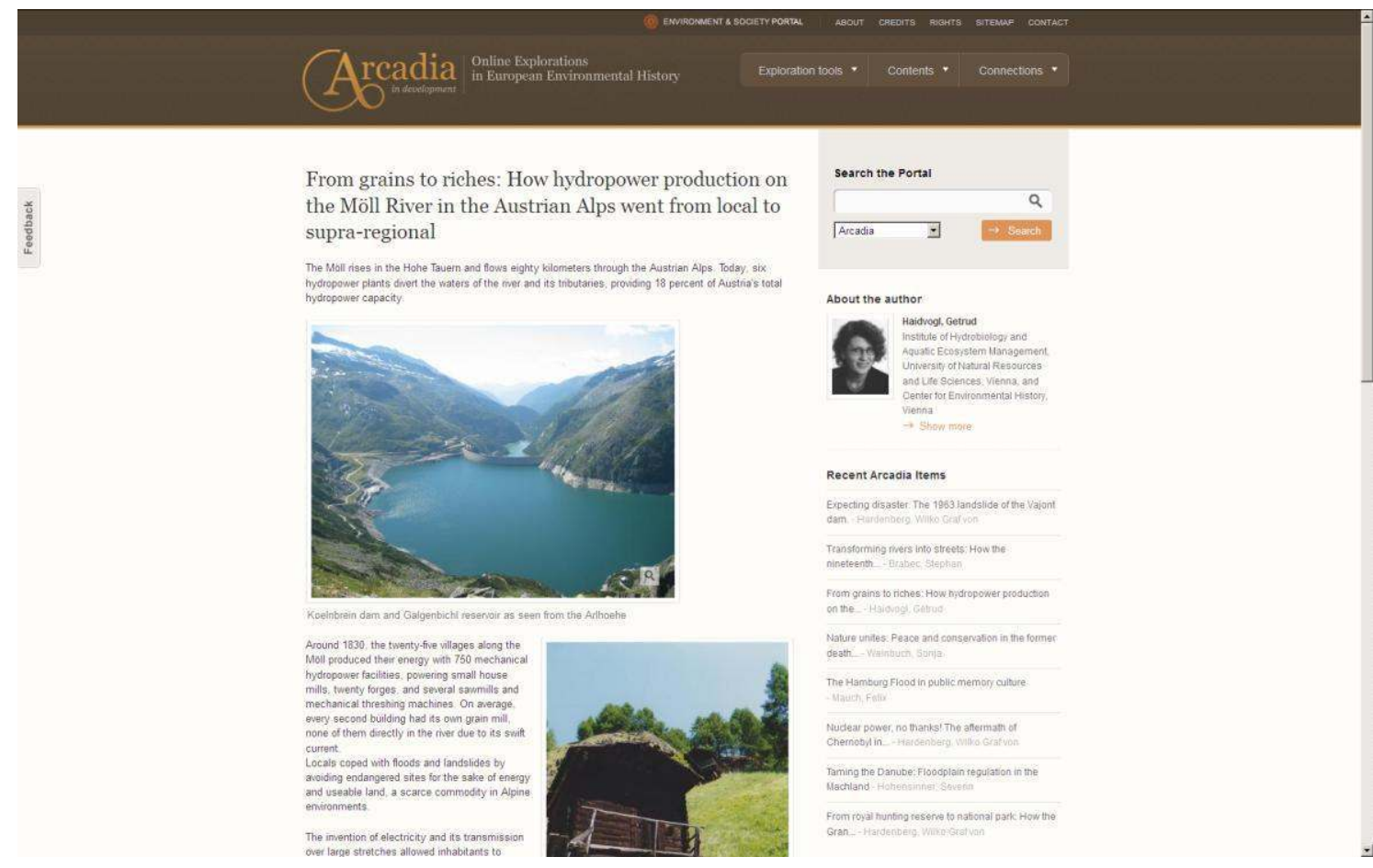

Le mostre virtuali (exhibitions) del portale, sviluppate in cooperazione con importanti istituzioni internazionali, offriranno poi uno sguardo dettagliato ed esaustivo su alcuni temi specifici. Tra le mostre pubblicate e in fase avanzata di produzione si contano sguardi critici sulla promozione e la trasformazione del paesaggio lungo la linea ferroviaria Chicago, Burlington, Quincy negli Stati Uniti (in cooperazione con la Newberry Library), sui diari inediti dell'esploratore polare tedesco Alfred Wegener (assieme al Deutsches Museum), sulla ricezione internazionale di un libro fondante del moderno movimento ambientalista, il Silent Spring di Rachel Carson (con la Beinecke Library della Yale University), e sulle diverse interpretazioni e traduzioni del concetto di wilderness in varie lingue europee (con l'Università di Zurigo).

\footnotetext{
9 URL: < http://www.environmentandsociety.org/arcadia > [consultato il 22 aprile 2012].

${ }^{10}$ URL: < http://www.eseh.org >[consultato il 22 aprile 2012].
} 


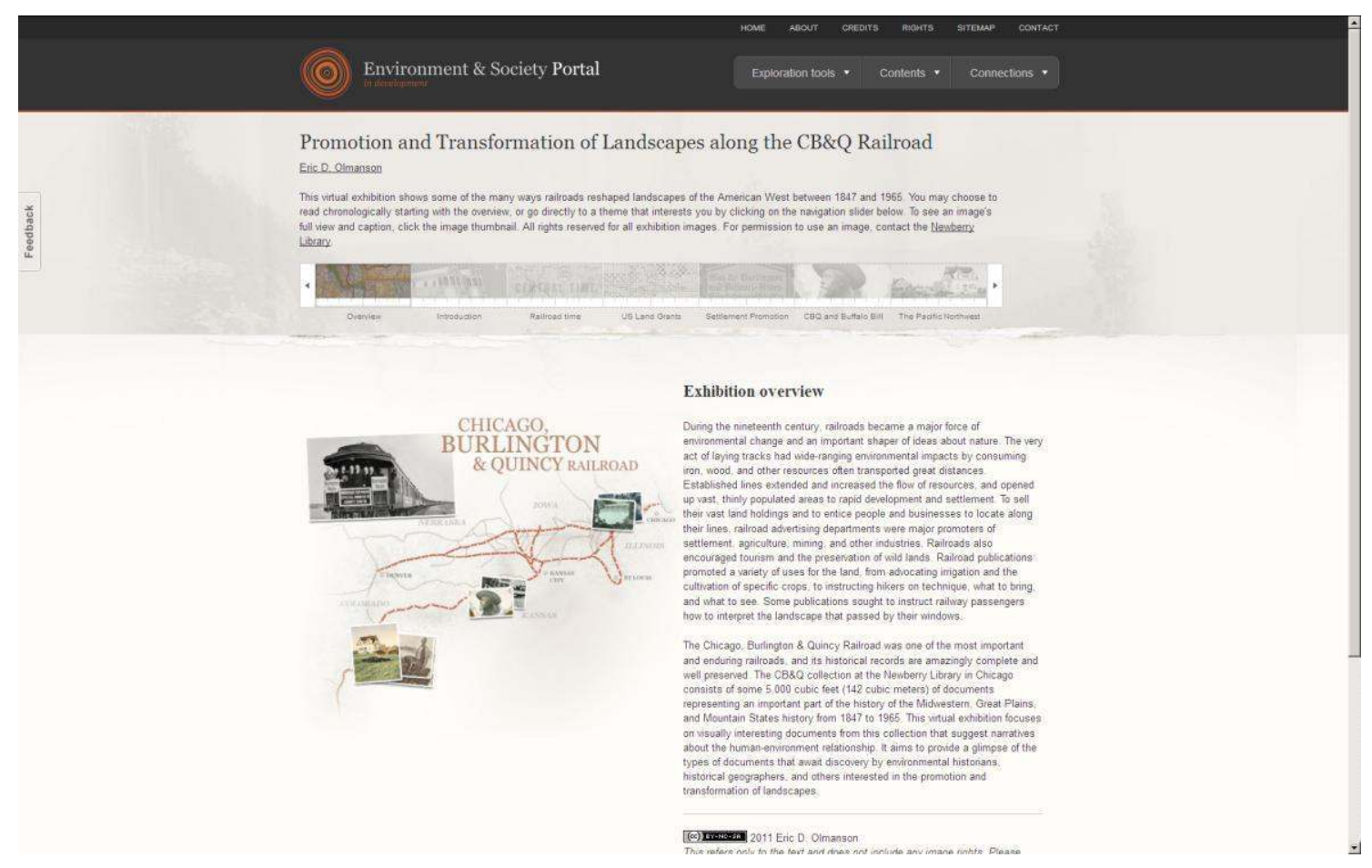

\subsection{Eterogeneità del posseduto ed elaborazione di percorsi di navigazione}

Una domanda che potrebbe sorgere è però come sia possibile costruire un portale che dia un impressione di coesione e di cui siano chiari gli obiettivi con materiale così eterogeneo. Inoltre, in fase di sviluppo ci siamo posti il problema di come stimolare costantemente gli utenti a procedere nella loro ricerca di nuovo materiale. Nel tentativo di rispondere ad entrambe queste questioni e permettere agli utenti di accedere ai contenuti da una pluralità di punti di vista il portale offre la possibilità di esplorare i contenuti in base a criteri geografici (map viewer), cronologici (timeline) e concettuali (keyword explorer). Sebbene il portale offra anche la tradizionale indicizzazione dei contenuti e la possibilità di svolgere ricerche full text questi tre strumenti di navigazione interconnessi rappresentano il suo tratto caratterizzante. L'obiettivo dell'adozione parallela di questi tre strumenti di ricerca e navigazione è di permettere al pubblico di creare percorsi di ricerca assolutamente personalizzati in modo che, intrecciando le possibilità offerte da strumenti così diversi, gli utenti possano sviluppare nuove idee di analisi e visualizzazione degli eventi storici o, anche, riflettere in maniera innovativa sulle tradizionali interpretazioni storiografiche. Inoltre, stiamo lavorando per permettere agli utenti di salvare questi percorsi di ricerca e condividerli o riutilizzarli a fini didattici. Riteniamo infatti che sia importante offrire la possibilità di 
diffondere non solo i contenuti e le informazioni, ma anche i processi attraverso i quali vi si è giunti. A tutti i contenuti, come vedremo in dettaglio più avanti sono stati attributi metadati geografici, temporali e concettuali che ne facilitano l'interconnessione e trasformano il database in una vera e propria esperienza di ricerca e scoperta. Ovviamente, strumenti come questi esistono anche altrove, ma raramente offrono un'esperienza di ricerca così completa e una tale, voluta, integrazione tra contenuti e strumenti informatici ${ }^{11}$. Inoltre, piuttosto che riutilizzare semplicemente approcci e strumenti già esistenti, abbiamo preferito rivedere i nostri presupposti relativamente ai modi in cui i contenuti possono essere categorizzati e rappresentati adattando o creando di conseguenza le necessarie tassonomie, l'aspetto grafico e le funzionalità.

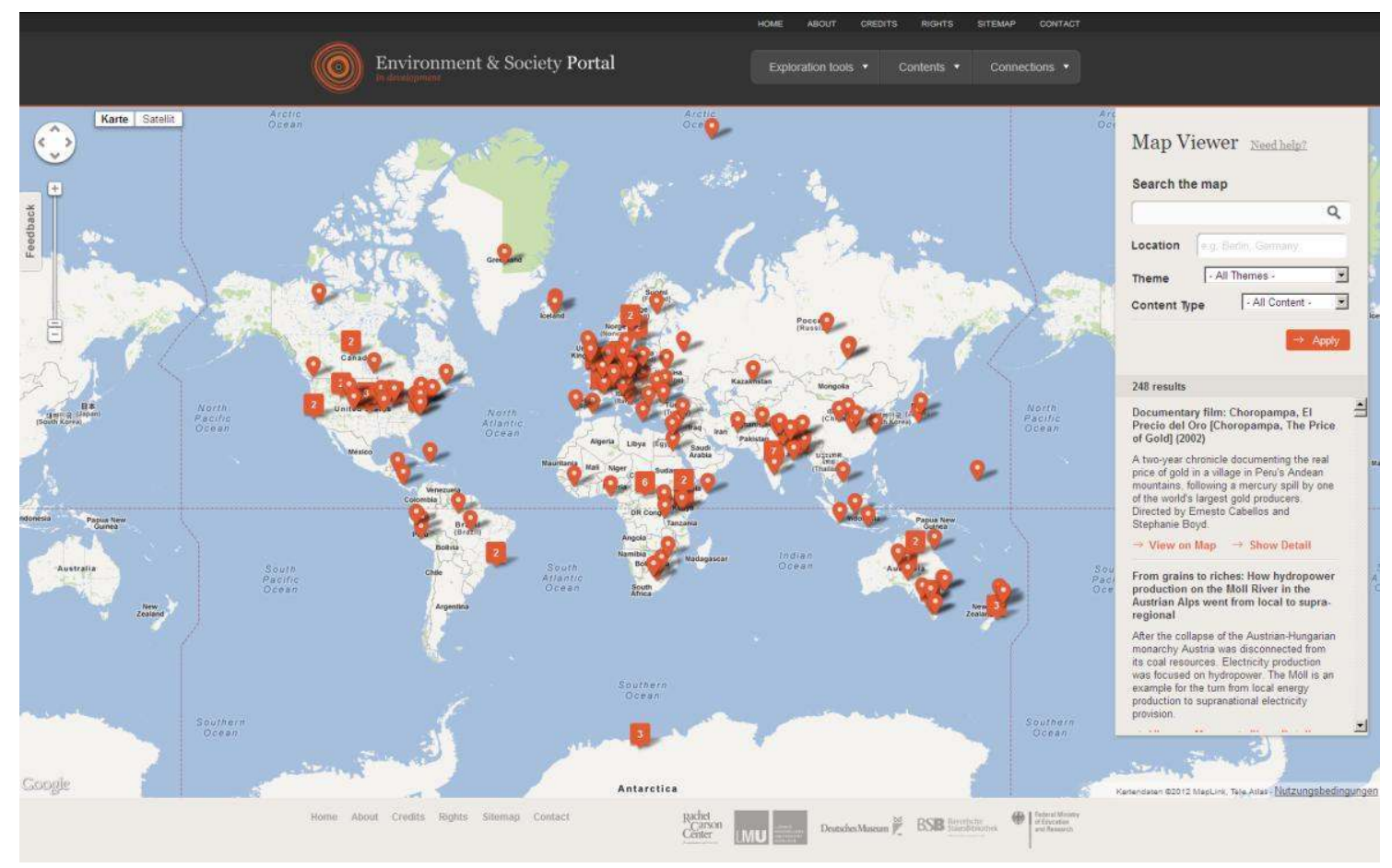

${ }^{11}$ Un interessante esempio di strumenti di visualizzazione di contenuti archivistici e bibliotecari su base geografica, cronologica e concettuale è offerto dal progetto ViewShare (della Library of Congress; URL: < http://viewshare.org > [consultato il 22 aprile 2012]. Si tratta però in questo caso di una suite di software, che possono essere utilizzati sia separatamente che congiuntamente, che hanno l'obiettivo di facilitare alle istituzioni culturali la presentazione in rete in maniera innovativa di collezioni già esistenti. Il portale, invece, ha come obiettivo, oltre a rendere la visualizzazione la più coinvolgente possibile, anche la creazione di una nuova collezione online espressamente dedicata alle environmental humanities. Anche europeana.eu, il portale europeo che intende raccogliere e presentare al pubblico in formato digitale i beni culturali del continente, offre del resto la possibilità di cercare i contenuti su base geografica e cronologica, usando rispettivamente OpenStreetMap - URL: < http://openstreetmap.org > [consultato il 22 aprile 2012] - e il software Timeline del progetto Simile del MIT di Boston, URL: < http://simile.mit.edu > [consultato il 22 aprile 2012]. 


\section{Obbiettivi e intenti dell'Environment \& Society Portal: la scelta degli strumenti}

L’intento originale del map viewer era di portare il cosiddetto spatial turn nell'ambito della visualizzazione di un database di informazione storico-ambientali. La nostra ambizione era dunque di combinare ricerca delle informazioni e analisi geografica delle stesse in un unico strumento in grado di far letteralmente vedere le connessioni spaziali tra diversi eventi e documenti contenuti nel nostro database. A livello di rappresentazione delle informazioni, la scelta più difficile e importante è stata quella di rappresentare solo punti geografici e non linee o aree. Questa non è solo una decisione di tipo pragmatico e tecnico, ma ha l'obiettivo di sottolineare il ruolo del nostro map viewer come indice dei contenuti, piuttosto che come rappresentazione cartografica tematica di dati coerenti. Non è infatti nelle intenzioni del portale sostituirsi o concorrere con gli esistenti sistemi informativi territoriali (GIS) o proporsi come catalogo di tutto ciò che esiste nel fin troppo vasto mondo delle environmental humanities. Eventualmente potranno essere gli utenti ad usare, in un secondo tempo, le informazioni che dovessero ritenere utili per costruire strumenti di questo genere su altre piattaforme o sui loro siti personali ${ }^{12}$. L'intento primario del portale è quello di far vedere spazialmente la distribuzione dei propri contenuti, in modo da facilitarne la ricerca in base a criteri geografici.

Una delle questioni che ci siamo posti in fase di sviluppo, sempre relativa al bisogno di rendere più facile il processo di ricerca, era su quali basi organizzare i metadati geografici del portale. Dopo aver considerato svariate alternative abbiamo deciso di adottare il dizionario geografico fortemente gerarchico, ma open-source, GeoNames ${ }^{13}$, utilizzato anche da altri grandi portali europei come europeana.eu. La scelta per la soluzione open-source non è stata dovuta solo a considerazioni di tipo economico, ma anche, se non soprattutto, al bisogno di poter adattare un dizionario pensato e sviluppato per l'oggi agli specifici bisogni della rappresentazione di informazioni storiche. Inoltre l'uso di un dizionario con una forte struttura gerarchica dà la possibilità di riflettere nella maniera più accurata possibile sulle complessità delle strutture amministrative esistenti, di facilitare - potenzialmente - il reperimento di

${ }^{12} \mathrm{E}$ per facilitare il riutilizzo dei dati in altri applicativi è allo studio anche la possibilità di offrire l'accesso ai dati anche attraverso una cosiddetta API (o interfaccia di programmazione). In questo modo eventuali utenti tecnologicamente avanzati potranno usare i metadati offerti dal portale per costruire rappresentazioni, visualizzazioni e processi di analisi personalizzati su specifici sottoinsiemi dei contenuti, magari incrociandoli anche con i dati ricavati da altri database presenti in rete.

13 URL: < http://geonames.org > [consultato il 22 aprile 2012]- 
informazioni nested (per esempio mostrando i risultati per un determinato villaggio anche fra i risultati del paese di cui fa parte), e di permettere una maggiore futura interoperabilità con altri siti.

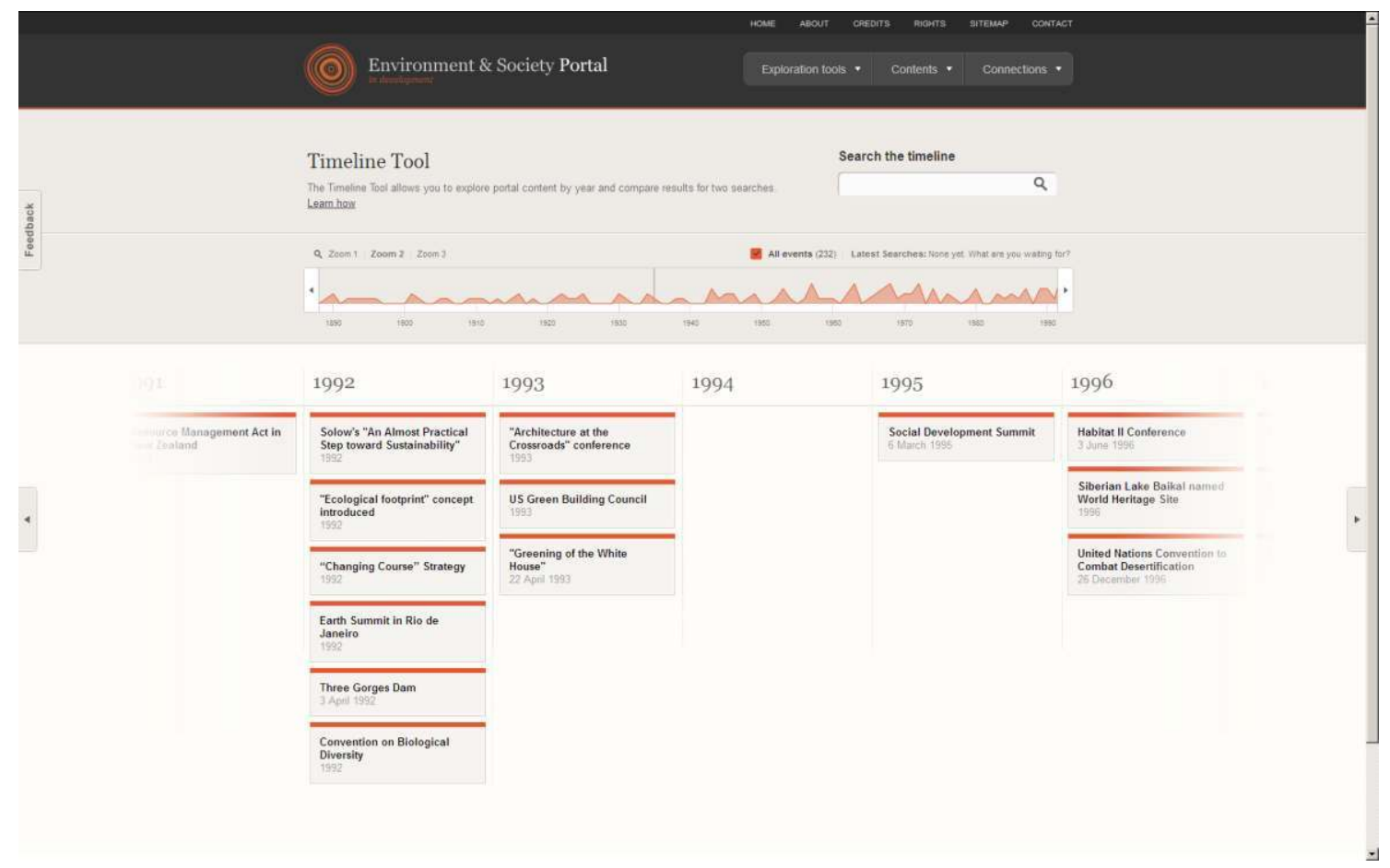

La timeline permette agli utenti di navigare lungo l'asse temporale, focalizzarci su periodi specifici e comparare su un grafico interattivo i risultati di due ricerche per parola chiave. In questo caso una delle decisioni più difficili, presa in un certo senso in parallelo alle decisioni prese per il map viewer, è stata quella di limitare i metadati temporali a singole date, interpretate come punti nel flusso del tempo, escludendo la rappresentazione o forzando l'approssimazione di periodi, ere o date fuzzy. Per eventi che coprono un arco temporale più ampio (per esempio la vita di un individuo o una guerra) nella maggior parte dei casi abbiamo scelto di rappresentare solo la data (anche approssimativa) d’inizio. Sebbene storiograficamente questa scelta abbia degli ovvi limiti, essa ha però anche dei risvolti positivi, permettendo una migliore e più chiara rappresentazione grafica del flusso temporale e dei singoli eventi. Inoltre ci siamo premurati, in fase di studio, di capire quale potesse essere il modo migliore per distinguere tra risultati per lo stesso anno e per rappresentare graficamente il corso del tempo. Come nel caso del map viewer era poi importante per noi sottolineare il fatto che anche la timeline è sostanzialmente un indice dei contenuti, piuttosto che una cronologia onnicomprensiva, come quelle che spesso si trovano nelle appendici dei libri di testo. Infine, la decisione di rappresentare la quantità di contenuti per ogni anno come picchi in un grafico potrebbe essere una delle scelte grafico-rappresentazionali 
più problematiche e più a rischio di essere soggette a critiche. Al momento però, soprattutto per motivi tecnici e grafici, non siamo ancora stati in grado di sviluppare una modalità alternativa che abbia la stessa efficacia nel dare velocemente all'utente una prima impressione della quantità di contenuti disponibili per una certa data o per un determinato periodo.

Mentre la mappa e la cronologia permettono agli utenti di confrontare rispettivamente i risultati in base a criteri geografici e temporali, il keyword explorer offre la possibilità di navigare i contenuti per temi, argomenti e concetti, in maniera funzionalmente simile al vecchio servizio di Google Wonder Wheel. Nello specifico il nostro strumento per la navigazione concettuale permette di affinare una determinata ricerca in base al numero di ricorrenze di una determinata parola chiave (tag) tra quelle assegnate dai redattori del progetto nei contenuti del portale. In futuro sarà anche possibile svolgere ricerche più libere ed esplorative in cui le parole chiave saranno collegate tra loro senza limitare il numero di risultati in base ai tag o ai temi scelti in precedenza. Per facilitare questo processo di ricerca concettuale abbiamo scelto di adottare e sviluppare autonomamente un vocabolario controllato non gerarchico di parole chiave rilevanti per le environmental humanities, invece di imporre a priori una struttura gerarchica predeterminata ai temi e ai $\operatorname{tag}^{14}$. Questo significa che l'esperienza di ricerca concettuale potrà modificarsi sostanzialmente con l'aggiunta di nuovi contenuti al portale, producendo al tempo stesso risposte più precise e modulate sulle ricerche degli utenti e un'esperienza più interattiva in cui i contenuti vengono raggruppati tematicamente sempre in maniera diversa.

\footnotetext{
${ }_{14}$ Abbiamo preferito limitare la scelta a un set limitato di parole chiave, circa 400 al momento, anziché adottare una politica di tag liberi come quella usata, per esempio, dai principali siti di condivisione di fotografie come flickr.com - URL: < http://www.flickr.com > [consultato il 22 aprile 2012] - o pinterest.com - URL: < http://www.pinterest.com > [consultato il 22 aprile 2012] - in modo da evitare che attraverso l'uso di sinonimi o per colpa di errori di battitura alcune connessioni tra i contenuti andassero perse. Dover far riferimento a un numero limitato di tag costringe il redattore a fare scelte consapevoli riguardo a quali di questi utilizzare.
} 


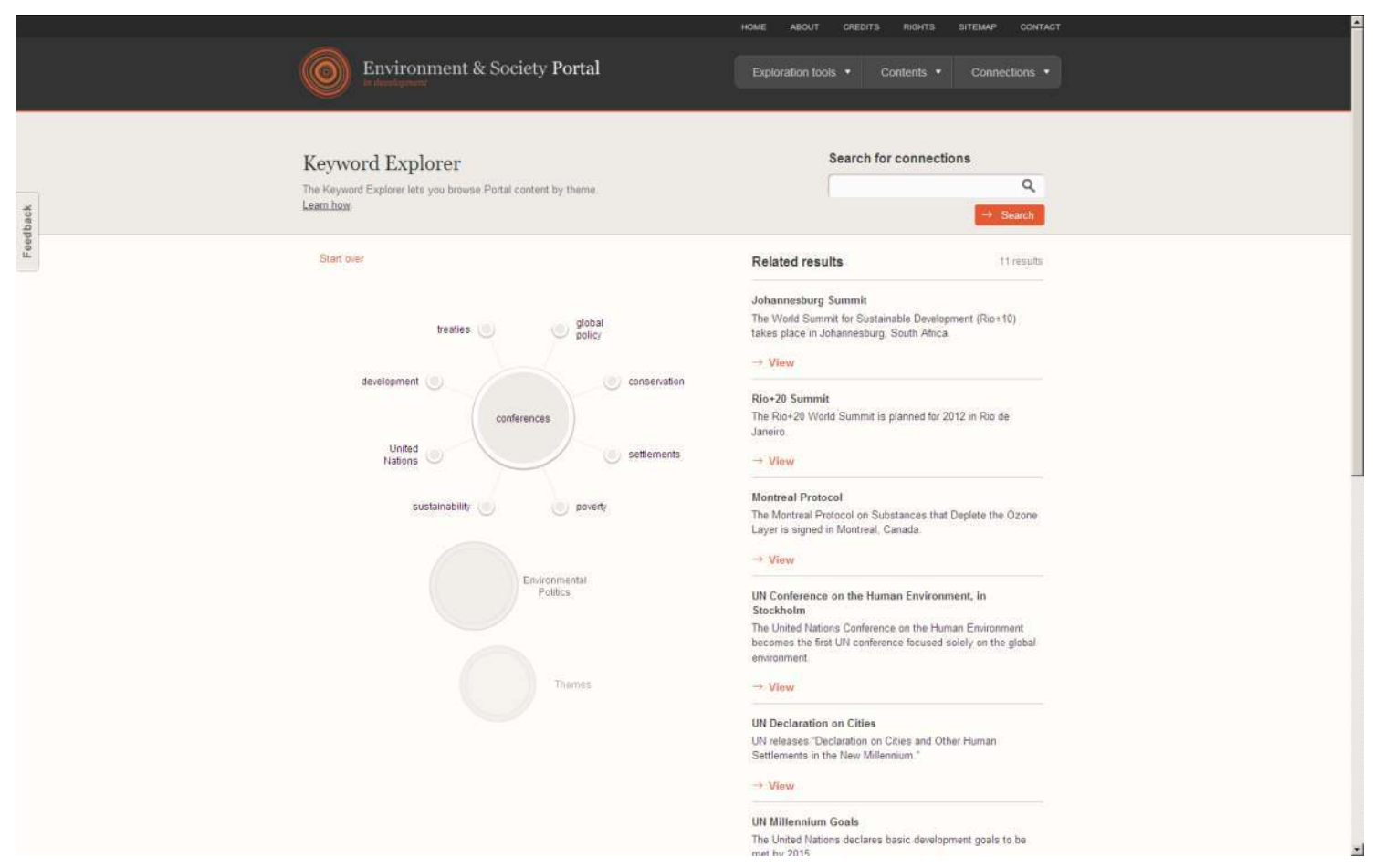

Le ragioni per cui per il map viewer è stato scelto un dizionario controllato gerarchico, mentre per la ricerca concettuale si è preferito fare a meno di strutture forti vanno ricercate nelle profonde differenze implicite alle due diverse tassonomie. Mentre i luoghi geografici sono quasi naturalmente organizzati su base gerarchica, soprattutto per quante attiene la loro collocazione amministrativa (per esempio, semplificando, stati-regioni-città), è molto più difficile costruire gerarchie soddisfacenti per i concetti. Spesso, infatti, questi ultimi non sono legati in maniera univoca ad un solo gruppo tematico (per esempio l'idea di canale può riferirsi sia al suo uso per il trasporto che al suo uso agricolo, rendendo poco sensato forzare il suo inserimento in uno solo dei due temi).

Ogni strumento di navigazione ha però dei limiti intrinseci rispetto ai modi in cui i dati e i problemi storici possono essere contestualizzati e rappresentati. Incrociando i risultati di strumenti diversi è quindi necessario porsi costantemente domande sulle implicazioni a lungo termine della loro interazione. La speranza è che la possibilità di far interagire strumenti diversi possa sopperire a questi specifici limiti e ricreare la citata serendipità intrinseca alla ricerca umanistica, e che le domande che ci siamo posti e le soluzioni che abbiamo tentato di trovare nel corso del lavoro di creazione dell'Environment \& Society Portal possano offrire utili prospettive sulle complesse considerazione ontologiche ed epistemologiche che si nascondono dietro alle semplici interfacce degli strumenti di navigazione. Prospettive che, magari, possano risultare 
utili ad altri soggetti interessati a mettere in piedi un portale umanistico e che si stiano chiedendo quali strumenti di ricerca e navigazione offrire ai propri utenti potenziali.

Un'impresa come il portale, che si propone al tempo stesso come enciclopedia, catalogo di fonti primarie e secondarie e museo virtuale, richiede un forte impegno nel campo dello sviluppo tecnico-informatico, in quello della raccolta e standardizzazione delle informazioni e anche in quello, più generale, della ricerca storiografica ed umanistica volta a giustificare le scelte effettuate. L'obiettivo a lungo termine è che un tale investimento possa avere effettivi e positivi riflessi sulla società globale, sia dal punto di vista di un aumento delle informazioni storiche e culturali disponibili riguardo all'attuale crisi ambientale, sia, potenzialmente, mettendo a disposizione nuovi strumenti informatici nel campo della divulgazione delle scienze umane in generale. Abbiamo qui citato in alcune note a piè di pagina delle soluzioni software open-source che possono essere utili ad integrare nei propri siti strumenti alternativi di visualizzazione dei contenuti, ma la nostra speranza è che in futuro saremo in grado di offrire, in quanto attore attivo nel campo della digital history, la tecnologia del portale, sotto forma di pacchetto informatico integrato nell'ambiente Drupal, anche ad altre istituzioni che abbiano intenzione di sviluppare portali tematici.

La prospettiva è che la possibilità degli utenti di svolgere ricerche e fare collegamenti tra i contenuti cresca esponenzialmente con il passare del tempo e con l'aumentare del materiale raccolto nel nostro database, al momento limitato a un piccolo starter set di circa trecento voci. Il piano di lavoro prevede che questa quantità sfiori quota mille, tra documenti, mostre virtuali e voci enciclopediche, entro la fine del 2012, per poi crescere ulteriormente negli anni a venire, aprendosi sempre di più al coinvolgimento degli utenti come contributori. La scelta di partire con pochi contenuti, puntando ad accrescerne il numero solo nel medio periodo, è stata presa intenzionalmente, in modo da avere il tempo necessario per testare al meglio gli aspetti tecnico-informatici e raccogliere un primo feedback dagli utenti che permetta di ricalibrare in corsa sia gli aspetti tecnici e le funzionalità offerte che la scelta dei contenuti e dei processi editoriali. 


\section{* Gli autori}

Wilko Graf von Hardenberg è uno storico ambientale e dottore di ricerca in geografia (Cambridge) che lavora come Digital Humanities Research Specialist presso il Rachel Carson Center.

URL: < http://studistorici.com/progett/autori/\#von-Hardenberg >

Kimberly Coulter ha ottenuto un dottorato di ricerca in geografia sulla produzione e distribuzione dei prodotti culturali (UW-Madison) ed è il Project Director dell'Environment \& Society Portal.

URL: < http://studistorici.com/progett/autori/\#Coulter >

\section{Per citare questo articolo:}

GRAF VON HARDENBERG, Wilko, COULTER, Kimberly, «Navigare la storia: considerazioni sulla creazione di un portale di storia ambientale», Diacronie. Studi di Storia Contemporanea: Digital History: la storia nell'era dell'accesso, 29/6/2012,

URL:< http://www.studistorici.com/2012/06/29/grafvonhardenberg-coulter_numero_10/ >

\section{Diacronie Studi di Storia Contemporanea 3 www.diacronie.it}

Risorsa digitale indipendente a carattere storiografico. Uscita trimestrale. redazione.diacronie@hotmail.it

Comitato di redazione: Marco Abram - Giampaolo Amodei - Jacopo Bassi - Luca Bufarale - Alessandro Cattunar - Alice De Rensis Barbara Galimberti - Deborah Paci - Fausto Pietrancosta - Matteo Tomasoni - Luca Zuccolo

Diritti: gli articoli di Diacronie. Studi di Storia Contemporanea sono pubblicati sotto licenza Creative Commons 2.5 Possono essere riprodotti a patto di non modificarne i contenuti e di non usarli per fini commerciali. La citazione di estratti è comunque sempre autorizzata, nei limiti previsti dalla legge. 